\title{
Study and Analysis of Nail Images of Patients
}

\author{
Sneha Gandhat \\ Dept.of Comp Engg. \\ PCCOE, Pune
}

\author{
A. D. Thakare \\ Dept.of Comp Engg. \\ PCCOE, Pune
}

\author{
Swati Avhad \\ Dept.of Comp Engg. \\ PCCOE, Pune
}

\author{
Nityash Bajpai \\ Dept.of Comp Engg. \\ PCCOE, Pune
}

\author{
Rohit Alawadhi \\ Dept.of Comp Engg. \\ PCCOE, Pune
}

\begin{abstract}
The fundamental way of disease detection in the human body using the nail image of human fingers and using the data from the image on the basis of nail colour is elaborated in this paper. In Traditional System there were doctors who could predict the diseases based on the nails but they required more time $\&$ also they got poor result. So to overcome that problem we designed a new system called Disease Detection System (DDS) which will give better result in less time. The system uses DIP and analysing techniques to identify such colours of nails. DDS increases accuracy of such observations of nails. DDS applies digital image processing techniques on input nail images to find the certain features in the image using MATLAB. By the crux knowledge of medical palmistry it analyses certain features in image and predicts probable disease. So with the help of DDS system we can detect the disease in its early stage hence the cost can be reduced for the further treatment. As the disease is detected in the early stage so the diagnosis becomes easy for the particular disease.
\end{abstract}

\section{Keywords}

Nail Color and Diseases, (Disease Detection System) DDS, Digital Image Processing Technique

\section{INTRODUCTION}

Medical science has advanced in many ways and has -ed different methods for finding of diseases in human body and one of the various ways to identify disease is through nails of the human palm. There are numerous testing ways of diagnosis of disease which are used by medical practitioners using pathological tests as basis. Mostly these involve taking blood samples which are quite painful and require the patient physically. The proposed system that is being developed is focused on image recognition based on color analysis. In the field of healthcare, study of human nail has its own significance. Many diseases can be diagnosed by scrutinizing nails of the hand. As human eyes have limitation in distinction in color.

This proposed system has algorithm which will automatically extract nail area and scrutinize this nail part for disease detection based on color of nail. The pathological are cumbersome to perform as their results can vary and these tests can take more than 24 hours by the examiner to establish name of the disease. Even it requires patient to be present for test, but our proposed system requires only the image of patients' hand that can be obtained easily and it's not hard to perform [1]. For examination full steps should be taken.

Image of the nail must be taken. Extract the area to be examined. Examine the nail color. Compare the nail with the pre-defined range. Note the condition of the nail. Reach on a conclusion and specify the diagnosed disease.

This paper presents an approach towards diagnosis of diseases based on Medical Palmistry[ 2]. DDS (Disease Detection System) increases accuracy of such observations of nails. Human nails are also reflects some diseases. So by extracting the colour features from the human nails we can analyze the diseases like Diabetes and Jaundice.

This paper describes DDS as an application of digital image processing and analysis technique[3]. This can be useful in healthcare domain to predict diseases like Diabetes \& Jaundice from nails for human being. The images of human nail give as an input to the system. Then, system applies digital image processing techniques on input images to identify certain features in the image using MATLAB. By using knowledge base of medical palmistry it analyzes certain features in image and predicts probable disease.

\section{LITERATURE REVIEW}

This section gives idea about existing decision support systems in medical science and prior work done in the area of digital image processing for medical science domain.

\subsection{Nail Color Analysis: Disease Symptoms}

Blue nail indicates: Myocardial infarction, Hypotension, shock, Hypocalcaemia, severe infection. (Figure 2:a)[4]

Nails pitting indicates: Psoriasis, Alopecia areata, Eczema and Lichen planus. (Figure 2:b)[4]

White nail indicates: Anemia, Chemotherapy, Renal failure, Cirrhosis, Diabetes mellitus, Hereditary (rare)(Figure 2:c).[4]

Red nail indicates: Polycythemia (dark), Systemic lupus erythematosus, Carbon monoxide (cherry red), Angioma, Malnutrition (Figure 2: d) [4].

Yellow nail indicates: Diabetes mellitus, Amyloidosis, Median/ulnar nerve injury, Thermal injury, Jaundice 


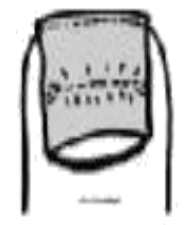

(1)

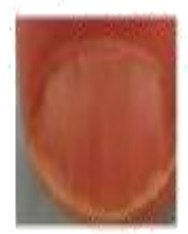

(d)

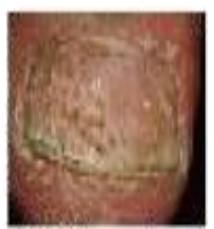

(b)

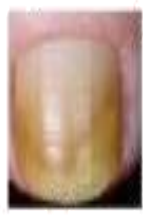

(1)

11

Fig. 1 Images of Nails

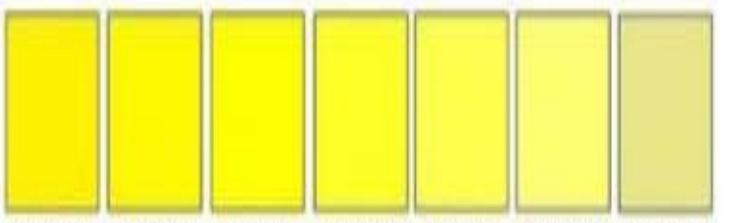

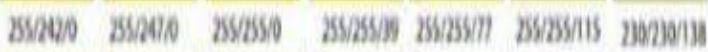

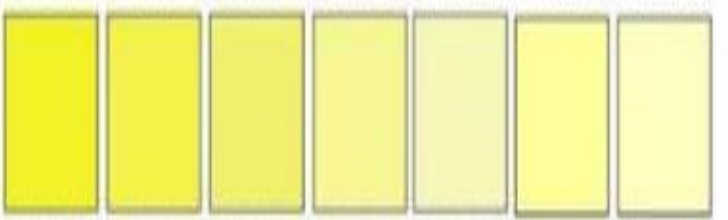

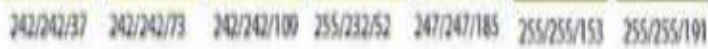

Fig. 2 Samples of Yellow Color Palette[5]

\section{PROPOSED SYSTEM}

In Traditional System, doctors can predict diseases by analyzing human nails because Nail colors are changing, these changes are related to physical condition changes caused by diseases or psychological and environmental factors but they require more time for that \& also they get less efficient result.So with the help of proposed system doctors can predict diseases by analyzing human nails because different nails colors can be indicate different diseases.

A set of methods or principles or rules for regulating the process is called Methodology. The Proposed Model has four major steps:

- Input to the model: Scanning the image containing fingers, back side of palm region in appropriate brightness.

- Extraction of the cropped Nail region from the entire Palm: Applying segmentation method on the image to extract the nails region from the entire image.

- Analysis of the Nail region, Color: The segmented nail image is now being processed on the basis of color and we will determine the health of the nail by comparing the segmented image with the dataset present.
- Disease Prediction using Knowledge base: The result will be generated on the basis of analysis and the disease would be predicted if present.

Fig.1 shows the system architecture of the DDS system.

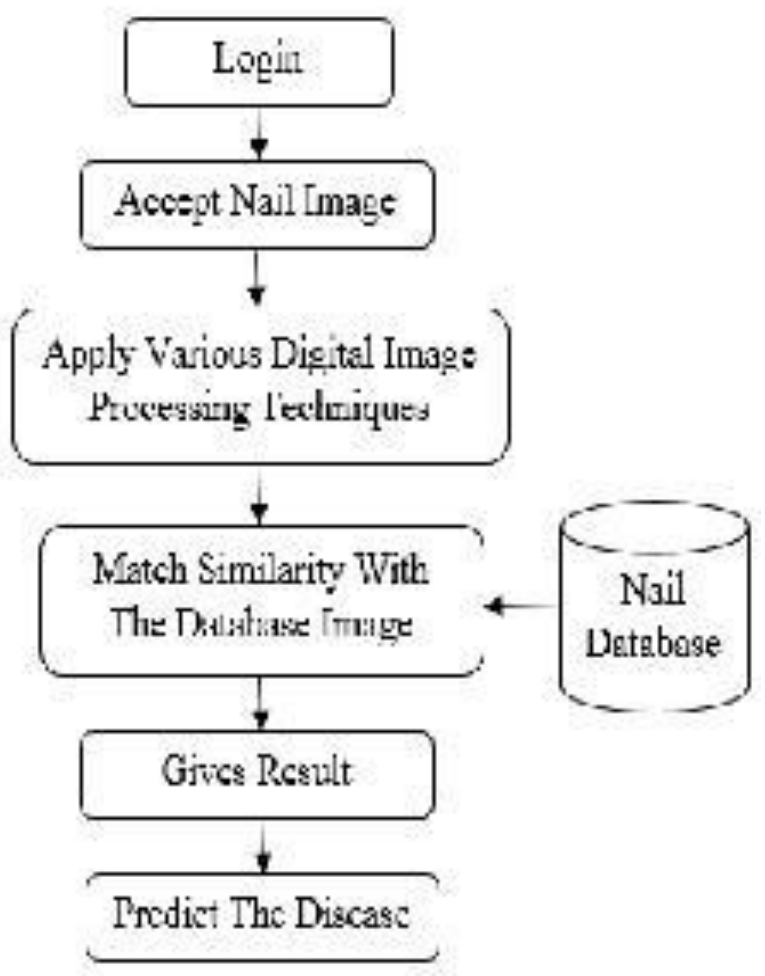

Fig. 3 DDS Architecture

In proposed system,user has to login to the system in order to get the prediction of a particular disease So nail images as an input to the system. Then further processing is done on the query image of nails.

\subsection{Nail Image Processing}

This section will show methods and techniques of several images preprocessing in order to process the nail image.

\subsubsection{Feature Extraction}

Feature extraction is one of the stage of our system to extract the colour feature from the image[6].After that applied Haar transforms to generate feature vector of these images.Feature vector generated are stored in a table which we call feature vector that is useful to reduce the size of feature vector for feature comparison process. Feature extraction helps to improve the performance of the system and improve GAR of our system.

\subsubsection{Various similarity measures}

Similarity measure are used to find similarity between two images like A and B[7]. They take on large values for similar objects and either zero or a negative value for very dissimilar objects is a commonly used similarity measure for real-valued vectors, used in information retrieval to score the similarity of documents in the vector space model and in machine learning model [8].The system uses similarity measures like Mean Square Error[9] and Euclidian Distance[10][11]. 


$$
\begin{aligned}
& \mathrm{ABS}=\frac{1}{m+n}\left[A^{y}-B^{y}\right] \\
& \mathrm{MSE}=\frac{1}{m+n}[A-B]^{2}
\end{aligned}
$$

Taxial Distance $=\sum_{n} \| A-B \mid$

Square Chord $=\Sigma_{n}(\sqrt{A}-\sqrt{B})^{2}$

Canberra $=\sum_{i=1}^{n} \frac{(A-B)}{A+B}$

Chebyshave $=\max (\|A-B\|)$

Eucledian Distance $=\sqrt{\sum_{n}(\|A-B\|)^{2}}$

Manhattan $=\sum_{i=1}^{n}\|A\| \hat{i}\|-B\| i\|\|$

$$
\left.\left.\operatorname{Cosine}(x i, y i)=\sum x i y i /\left(\sqrt{\sum(x i)}\right)^{2} \sqrt{\sum(y i)}\right)^{2}\right)
$$

\subsubsection{Performance Comparison}

Performance comparisons is done using genuine acceptance ratio (GAR) and overall accuracy measurement of the images.Calculate the accuracy of total images present in dataset with the help of feature vector.
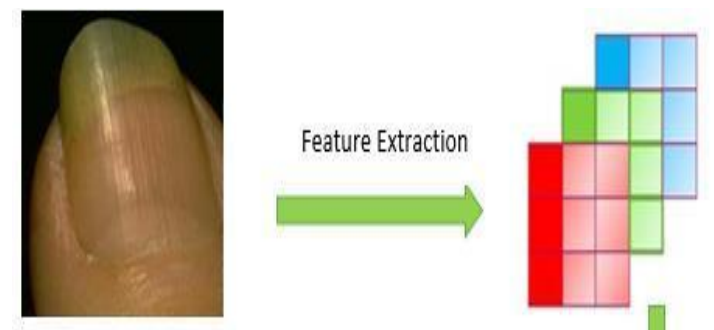

Transform

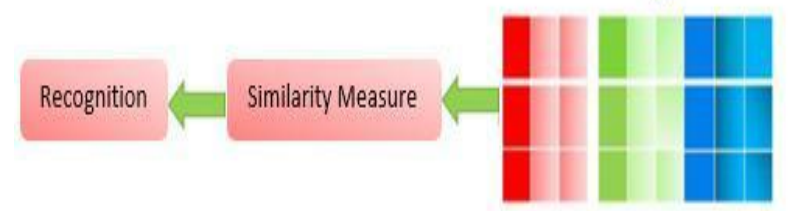

Fig. 4 Processing Steps of Nail Image

\section{Steps to process the Nail image}

- Read all images from dataset.

- Extract Colour Feature from that images.

- Apply Haar Transform Matrix on that image[12].

- Generate query feature vector.

- Apply Row Mean to reduce Feature vector.

- Compare a query feature vector stored in template dataset using similarity measure like MSE, absolute difference.

- Find out matching store and calculate GAR.

- Display Result.

\section{RESULTS}

Disease Detection System (DDS) detects diseases like Juandice and Diabetes by with the help of nail color.Nail images of patients who is having the juandice and diabetes disease are stored in the database as Training images.So Fig. 4 and Fig. 5 shows the matching result of Tranining image with the Test image of nail. Which predicts the diseases like Diabetes and Juandice respectively.

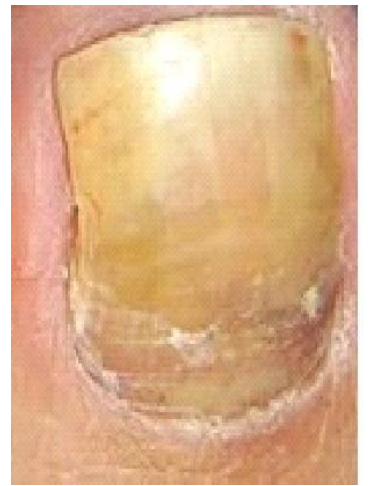

Trained image for Diabetes

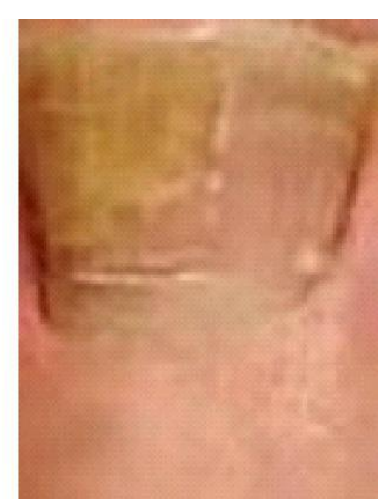

Test image
Fig. 5 Image matching Result for Prediction of Diabetes Disease

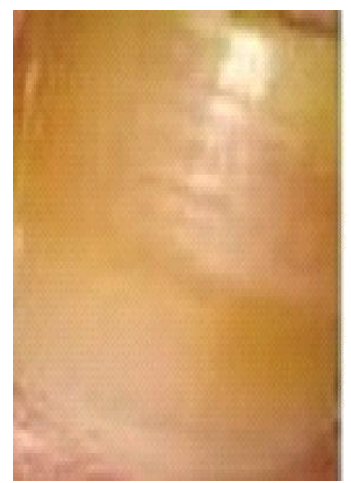

Trained image for Juandice

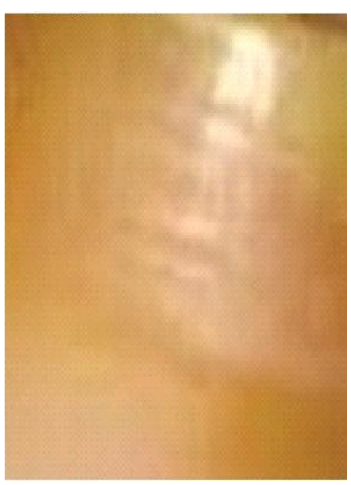

Test image
Fig. 6 Image matching Result for Prediction of Juandice Disease

\section{CONCLUSION}

Health is a critical aspect for Human life. Identification of human health conditions and accurately predicting the symptoms of the diseases is useful work for the society. For these a new system is designed based on DIP, medical palmistry and nail-color analysis. Nail colors are used for the disease detection. It helps in recognizing disease of the person and minimize the cost of the diagnosis of diseases. The detection system makes easy for doctors to give correct treatment to patients. The system can be made better with increased number of image samples. The future scope includes making the refining of the expectant results via the image.

\section{REFERENCES}

[1] M. Young, Technical "The Writer'sMill Handbook", Valley, CA: University Science, (1989).

[2] Hardik Pandit and Dipti Shah, "Decision Support System for Healthcare based on Medica Palmistry" Presented in ICISD-2011, GCET Engineering College, Vallabh Vidyanagar. 
[3] OVERVIEW OF IMAGE PROCESSING K.M.M. Rao*,Deputy Director,NRSA,Hyderabad-500 037

[4] M. E. Williams,Finger Nails: "ExaminingWhenthe evaluating presenting symptoms in elderly Patients", Accessed From:www.medscape.com, Accessed on: [22/12/2014], (2009).

[5] Nail Color and Texture Analysis for Disease Detection Vipra Sharma and Manoj Ramaiya International Journal of Bio-Science and Bio-Technology Vol.7, No.5 (2015 $\mathrm{Pp}$ $.351-358$ http://dx.doi.org/10.14257/ijbsbt.2015.7.5.34.

[6] Image Specific Feature Similarities Ido Omer and Michael Werman School of Computer Science, The Hebrew University of Jerusalem 91904 Jerusalem, Israel \{idom,werman\}@cs.huji.ac.il

[7] M.B. Waldron, S. Kim, "Isolated ASL sign recognition system for deaf persons" IEEE Trans. Rehabilitation Eng. 3(3) (1995) 261-271.

[8] Similarity Meaasures .pdf
[9] A New Metric for Quality Assessment of Digital Images Based on Weighted-Mean Square Error1 Proceedings of SPIE, vol. 4875, 2002 Kaiwen Zhang, Shuozhong Wang, and Xinpen Zhang School of Communication and Information Engineering, Shanghai University

[10] On the Euclidean Distance of Zhangt, Jufu Fengt †Center School of Electronics Engineering and Computer Science, Peking University, Beijing, P.R.China $\uparrow\{$ wanglw, zhangyan, fjf $\} @$ cis.

[11] Euclidean Distance Mapping PER-ERIK DANIELSSON Department of ElectricalE ngineering, Linkoping University, Linkoping \$-581 83, Sweden Received June 5, 1979; revised September 28, 1979; accepted February 6,1980

[12] The Haar-Wavelet Transform in Digital Image Processing: Its Status and Achievements Piotr Porwik, Agnieszka Lisowska Institute of Informatics, University of Silesia, ul. B edzin'ska 39, 41-200 Sosnowiec, Poland e-mail: porwik@us.edu.pl Institute of Mathematics, University of Silesia, ul. Bankowa 14, 40-007 Katowice, Poland e-mail: alisow@ux2.math.us.edu.pl 